DÉCOLONISATION ET CONSTRUCTION NATIONALE AFRIQUE, ASIE ET QUÉBEC

TITRE: LES MÉMOIRES DU GÉNOCIDE DE 1904 DANS LE SUD-OUEST AFRICAIN ALLEMAND Auteur(s): Estelle Bourbeau, Université de SHerbrooke

Publication: Décolonisation et construction nationale: Afrique, Asie et Québec

PAGE: 135-154.

Directeurs: PATrick Dramé, PAScal SCALlon-Chouinard et Françoise Nozati

ÉditeuR: LES ÉdITIONS DE L'UNIVERSITÉ DE SHERBROOKE, 2016.

ISBN: 978-2-7622-0353-0

URI: HTTP://HDL.HANDLE.NET/11143/8764

DOI: HTTP://DX.DOI.ORG/10.17118/11143/8764 


\section{LES MÉMOIRES DU GÉNOCIDE DE 1904 DANS LE SUD-OUEST AFRICAIN ALLEMAND}

\section{Estelle Bourbeau}

Avec ses deux guerres mondiales et ses génocides, le XXe siècle est un " siècle de violence ${ }^{1}$ ", et il a laissé au sein de nombreuses collectivités une mémoire peuplée de traumatismes, mais aussi d'oubli. En effet, l'historiographie occidentale accrédite généralement la croyance largement répandue qui attribue la sinistre palme du premier génocide du XXe siècle au génocide arménien, perpétré dans l'Empire ottoman de 1915 à 1916². II s'agit là d'un bel exemple d'amnésie collective qui équivaut à tirer un trait sur plusieurs milliers de victimes. En effet, de 1904 à 1908, pas moins de 50000 membres du peuple herero ainsi que 6000 représentants du peuple nama connaissent une fin brutale alors que s'affirme la domination coloniale allemande. Le vide démographique ainsi créé va permettre aux colonisateurs allemands, présents depuis $1884^{3}$, d'affirmer leur présence en exploitant les terres utilisées auparavant par les Herero pour leur élevage bovin, faisant ainsi de l'Afrique allemande du Sud-ouest une véritable colonie de peuplement ${ }^{4}$. Sur les 65000 Hereros qui vivent sur le haut plateau namibien, 15000 seulement parviennent à échapper à la mort ${ }^{5}$.

Malgré ce lourd bilan, I'hécatombe dont les Herero sont victimes à l'aube du XX' siècle est un des génocides les plus méconnus, et ce particulièrement en Occident. Les raisons de cette méconnaissance sont multiples. Tout d'abord, le passé colonial allemand est encore peu visité, que ce soit par les historiens ou par la fiction. Ensuite, ceux qui s'intéressent effectivement à la question des crimes commis en sol africain auront beaucoup plus de facilité à s'informer au sujet de l'apartheid sud-africain et du génocide rwandais, événements fortement médiatisés.

1. Christian Coq, dir., Travail de mémoire 1914-1998. Une nécessité dans un siècle de violence, Paris, Autrement, 1999, p. 5.

2. Nadja Vuckovic, "Qui demande des réparations, et pour quels crimes? », dans Marc Ferro, dir., Le livre noir du colonialisme. XVk-XXk siècle: de l'extermination à la repentance, Paris, Robert Laffon, 2003, p. 773.

3. Ingolf Diener, Namibie, une histoire, un devenir, Paris, Karthala, 2000, p. 60.

4. Bernard Lugan, Histoire de l'Afrique du Sud de l'Antiquité à nos jours, Paris, Perrin, 1986, p. 239.

5. Peter Biles, «Unfinished Business for Namibia's Herero », BBC News (19 janvier 2006), [en ligne] http://news. bbc.co.uk/2/hi/africa/4623516.stm, page consultée le 19 novembre 2012. 
Pourtant, le génocide herero a de quoi frapper les esprits. Plus de deux décennies avant la Deuxième Guerre mondiale, il montre des ressemblances troublantes avec l'holocauste : extermination planifiée et systématisée, travail forcé et, surtout, présence de camps de concentration ${ }^{6}$. La Shoah étant la tentative d'éradication la plus emblématique du XXe siècle (à un tel point qu'on peut la qualifier, au niveau mémoriel, de véritable « étalon de mesure de l'horreur $\left.^{7} »\right)$, on serait porté à croire qu'un parallèle aussi significatif aurait garanti la popularité du génocide herero. Cependant, les traces du génocide ne subsistent que dans la mémoire collective des descendants des herero vivant encore en Namibie et qui demandent réparation ${ }^{8}$

Par conséquent, et particulièrement compte tenu du caractère des évènements, il est nécessaire de se poser la question suivante : comment se construisent les modalités de l'oubli et de la mémoire chez les Namibiens et les Allemands, et comment s'articule leur rencontre? ॥ s'agit d'une question très large, à laquelle il est nécessaire de répondre en trois temps. Tout d'abord, pour les Allemands, la mémoire du génocide herero se présente comme une amnésie, car elle contredit la mémoire actuellement entretenue au sujet du colonialisme et de l'holocauste. En effet, le tissu mémoriel allemand a tendance à se considérer comme libre de la tâche du colonialisme ${ }^{9}$, perception qui serait remise en question si un débat public s'ouvrait sur le problème herero. Quant aux Herero, la mémoire du génocide s'est transmise au sein de la communauté sans intégration à l'histoire officielle, mais s'est extériorisée vers l'ensemble de la Namibie avec la commémoration du centenaire des évènements ${ }^{10}$. Enfın, la rencontre de ces deux mémoires provoque un difficile dialogue entre l'Allemagne, qui tente de voir le génocide pardonné avant de l'avoir assumé, et les Herero, qui demandent réparation et reconnaissance dans une perspective de justice sociale ${ }^{11}$.

La première partie de cette analyse, qui portera sur les causes et le déroulement du génocide, sera abordée dans une perspective d'histoire-récit. En effet, puisque le génocide herero est largement méconnu, il importe dans un premier temps d'en rappeler les évènements majeurs. Cette synthèse permet aussi de mieux appréhender les enjeux de la mémoire du massacre de 1904. Dans la deuxième partie, les mémoires allemande et herero du génocide seront analysées à tour de rôle : chez les premiers, c'est un oubli quasi total qui peut être constaté, alors qu'une mémoire centrée sur la victimisation caractérise les seconds. Cette

6. Robert Gerwarth et Stephan Malinowski, «L'antichambre de l'holocauste? À propos du débat sur les violences coloniales et l'extermination nazie », Vingtième siècle. Revue d'histoire, vol. 3, nº 99 (2008), p. 144.

7. Emmanuel Droit, "Le Goulag contre la Shoah : Mémoires officielles et cultures mémorielles dans l'Europe élargie », Vingtième Siècle. Revue d'histoire, nº 94 (avril-juin 2007), p. 119.

8. Nadja Vuckovic, op. cit., p. 777.

9. Johannes Wendt, «L'anamnèse du colonialisme allemand », L'homme et la société, n 175 (2001), p. 60.

10. David Olusoga, Namibia: Genocide and the Second Reich, Enregistrement vidéo, BBC Bristol, Bristol, 2005, 1 vidéodisque : 58 minutes, son, coul., DVD.

11. Ibid. 
section se basera sur la riche historiographie consacrée aux productions mémorielles allemandes et namibiennes. Enfin dans la troisième partie du texte, nous étudierons la question de la rencontre et du dialogue de ces deux mémoires à travers les démarches effectuées par les descendants des survivants pour obtenir réparation. Ces démarches, qui débutent au début des années $2000^{12}$, ont laissé des traces médiatiques dans les journaux et sur Internet.

\section{LE GÉNOCIDE HERERO : UN RÉCIT MÉCONNU}

La complexité de la question mémorielle entourant le génocide herero est indissociable de la prise en compte d'un certain nombre d'éléments clefs. Nous étudierons tout d'abord le contexte namibien au moment du déclenchement du génocide, puis le déroulement du génocide lui-même et finalement la situation des survivants à l'issue des événements.

\section{LA NAMIBIE À LA VEILLE dU GÉNOCIDE}

Durant la seconde moitié du XIXe siècle, les Herero sont un peuple acéphale et nomade dont la principale activité est l'élevage intensif de bovin ${ }^{13}$. Ils sont issus d'un schisme survenu au $\mathrm{XVl}$ e siècle au sein du peuple Mbandu, qui résidait alors dans l'actuel Botswana; les Herero, dont le nom signifie littéralement « ceux décidés » représentent la partie des Mbandu qui choisit de partir plus au Nord, en quête de plus verts pâturages ${ }^{14}$. L'identité herero précoloniale est ainsi profondément liée à la relation de l'individu avec le bétail, aussi bien au niveau matériel que spiritue ${ }^{15}$. Les immenses pâturages nécessaires à l'alimentation des bêtes sont par ailleurs «la propriété collective inaliénable de la tribu entière ${ }^{16}$. »

Les Nama, quant à eux, sont un des groupes Khoï-Khoï (aussi connus en Occident sous le nom de "hottentots ») originaires du Nord-Est africain ${ }^{17}$. Organisés en plusieurs chefferies indépendantes, ils pratiquent l'élevage à plus petite échelle que les Herero et entretiennent avec la terre et les bêtes des relations semblables aux leurs ${ }^{18}$. C'est graduellement que se fait l'introduction des Allemands dans l'espace herero et nama. Les marchands en sont les premiers ambassadeurs. L'un d'entre eux, Adolf Lüderitz, réussit en 1884 à obtenir la signature de contrats de protection et d'achat de terres à des chefs africains : ces documents

\section{Ibid.}

13. Ingolf Diener, op. cit., p. 45.

14. Ibid., p. 44.

15. Ibid., p. 45.

16. Ibid.

17. Ibid., p. 49.

18. Ibid., p. 48. 
permettent ensuite à Bismarck de revendiquer le territoire, baptisé Afrique allemande du Sud-ouest, lors du traité de Berlin en $1885^{19}$. À partir de ce moment, des colons allemands immigrent en Namibie pour tenter d'y exploiter la terre, les Herero se trouvant au départ encore en position de force ${ }^{20}$.

Cette situation allait toutefois être renversée : pour Bismarck, l'agrandissement de l'espace vital ou Lebensraum pour garantir l'épanouissement de colons allemands est une priorité capitale qui passe par l'établissement d'une colonie forte en Afrique ${ }^{21}$. Après des débuts peu satisfaisants sous la gouverne d'Heinrich Göring, c'est à Theodor Leutwein, gouverneur à partir de 1894, que revient la tâche de créer un environnement propice à l'épanouissement des colons allemands. Face à une situation d'infériorité numérique, Leutwein emploie des moyens politiques plutôt que militaires pour faire passer les ressources des mains des populations locales vers celles des colons : il sème la discorde parmi les différents groupes de façon à saboter le système d'alliances en place ${ }^{22}$. Aidé des Schütztruppe, petites troupes militaires présentes dès $1888^{23}$, Leutwein établit aussi un processus d'expropriation et de domination, profitant d'évènements comme la peste bovine de 1897 pour favoriser l'émigration des populations désespérées vers les mines d'Afrique du Sud ${ }^{24}$ et rafler leurs terres ${ }^{25}$. En 1903, Herero et Nama se retrouvent donc déjà en voie de devenir une force ouvrière prolétarisée ${ }^{26}$ et victime d'exactions brutales ${ }^{27}$ sous le joug conjugué des fermiers et des soldats ${ }^{28}$.

\section{VON TROTHA ET L'ORDRE D'EXTERMINATION}

Le statu quo n'allait cependant pas durer. Après une insurrection rapidement matée en 1896, Herero et Nama se mobilisent en 1904 dans l'espoir de freiner l'avancée allemande. Avec à sa tête Samuel Maharero, une force de 6000 hommes entre en guerre et attaque le poste de traite allemand d'Okahandja, tuant 123 colons avant d'entamer une reprise de contrôle du

19. Ibid.

20. David Olusoga, op. cit.

21. Ibid.

22. Ingolf Diener, op. cit., p. 97.

23. [s. a.], "A bloody history : Namibia's colonisation », BBC News (29 août 2001), [en ligne] http://news.bbc.co.uk/2/hi/africa/1514856.stm, page consultée le 10 décembre 2012.

24. Jan-Bart Gewald, «The Road of the Man called Love and the Sack of Sero: The Herero-German War and the Export of Herero Labour to the South African Rand », The Journal of African History, vol. 40, n 1 (1999), p. 22.

25. Ingolf Diener, op. cit., p. 103.

26. Ibid., p. 107.

27. Ibid., p. 110.

28. David Olusoga, op. cit. 
territoire ${ }^{29}$. C'est là que la situation bascule : Leutwein, dont les manœuvres diplomatiques sont jugées inefficaces dans la résolution du conflit, est remplacé par le général Lothar von Trotha $^{30}$. Von Trotha aborde la rébellion Herero-Nama dans une perspective de guerre raciale, envisageant dès son arrivée l'extermination totale de l'ennemi ${ }^{37}$. II ne s'agit pas, pour lui, d'une opération de pacification à l'endroit d'insurgés, mais bien d'une lutte entre deux peuples pour le droit d'exister ${ }^{32}$.

Les Herero, quant à eux, ont dans leur reconquête essuyé des pertes importantes et se considèrent vaincus; l'ensemble de leur peuple s'assemble à Waterberg, à la lisière du désert d'Omaheke, dans le but de négocier leur reddition ${ }^{33}$. C'est là que Von Trotha choisit de frapper, encerclant stratégiquement les Herero de façon à pousser ceux-ci à fuir dans le désert ${ }^{34}$. C'est après Waterberg que le fameux « Ordre dit d'extermination » de 1904 est donné par Von Trotha à ses troupes et au peuple herero:

Moi, le grand général des troupes allemandes, j'envoie ce message au peuple Herero [...] Tous les Herero doivent quitter ces terres [...] Tout Herero retrouvé à l'intérieur des frontières allemandes avec ou sans fusil, avec ou sans bétail, sera fusillé. Je ne recevrai plus de femmes ou d'enfants; je les renverrai à leur peuple. Je les fusillerai. Ceci est ma décision pour le peuple Herero ${ }^{35}$.

C'est cet ordre, associé avec l'idéologie de guerre raciale entretenue par Von Trotha, qui justifie l'appellation de « génocide herero » : selon la définition établie par Raphaël Lemkin et affinée par l'ONU, le génocide, qui peut prendre plusieurs formes, se caractérise par la mise en application systématique de mesures ayant pour but d'anéantir un groupe ${ }^{36}$. Cette intention, officialisée par Von Trotha à travers cet ordre, est même approuvée par le haut commandement militaire de Berlin, qui répond à la nouvelle de la fuite des Hereros dans le désert en affirmant que « [l]a lutte raciale en cours ne peut se conclure que par l'anéantissement d'une partie [...] de ce fait, l'intention du général Von Trotha peut être approuvée ${ }^{37}$. » Ainsi, malgré la capitulation des Herero, la guerre se poursuit avec l'acharnement d'une lutte d'extermination : beaucoup de Herero sont tués par balle, mais la majorité des victimes du génocide

29. Ibid.

30. Bernard Lugan, op. cit., p. 243.

31. Ingolf Diener, op. cit., p. 114.

32. David Olusoga, op. cit.

33. John liffe, Africans : the history of a continent, Cambridge, Cambridge University Press, 2007 (1995), p. 318.

34. David Olusoga, Namibia : Genocide and...

35. [s. a.], loc. cit., 29 août 2001.

36. Noor Akbar, "How should we define genocide? ", Human Dignity and Humiliation Studies, [en ligne]www.humiliationstudies.org, page consultée le 15 décembre 2012.

37. Ingolf Diener, op. cit., p. 115. 
périssent de faim et de soif dans le désert, une des stratégies de Von Trotha étant d'empoisonner les points d'eau ${ }^{38}$.

Cependant, lorsque l'ordre d'extermination parvient aux oreilles des populations européennes, le scandale éclate. Le Kaiser ordonne donc en 1905 la révocation de cet ordre : dorénavant, tous les Hereros capturés seront faits prisonniers plutôt que tués ${ }^{39}$. On voit alors s'ouvrir le second volet du génocide : celui des camps de concentration et des travaux forcés. Ces camps sont d'une dureté telle que la moitié des individus qui y sont internés meurent durant la première année. Au total, $45 \%$ de la population emprisonnée périt dans des camps comme celui de Swakopmund ${ }^{40}$. Les Herero prisonniers sont également loués à des entreprises qui les utilisent dans leurs travaux ${ }^{41}$.

Certaines d'entre elles, comme la Woermann, une compagnie d'import-export, font un tel usage de cette main-d'œuvre carcérale qu'elles dirigent leur propre camp de concentration. Notons par ailleurs que l'histoire voit apparaître dans le cadre de ce second volet du génocide le premier camp d'extermination à avoir vu le jour : il s'agit de Shark Island, petite île où est mise en place une tentative vite abandonnée d'éradiquer les $\mathrm{Nama}^{42}$, qui continuent de harceler les Allemands à travers des pratiques de guérilla jusqu'en 190943. Finalement, en 1908, les camps de concentration sont fermés et avec eux, le génocide herero-nama arrive à son terme. Les survivants sont, pour la plupart, vendus en tant qu'esclaves aux fermiers allemands ou parqués dans des réserves situées à la lisière du désert du Kalahari44.

UN PAYS TRAUMATISÉ

La fin du génocide signifie aussi, pour les survivants, la fin d'une ère. En effet, l'année 1908 marque la désintégration du tissu social herero et nama traditionnel, puisque les structures tribales sont démantelées et qu'il est dorénavant interdit aux Herero de se regrouper. De plus, non seulement les biens et le patrimoine foncier herero et nama sont-ils confisqués, mais il leur est désormais interdit de posséder du bétail, ce qui les prive de leur activité économique principale et du fondement de leur identité culturelle et spirituelle ${ }^{45}$. Les déplacements des

38. [s. a.], " Germany admits Namibia's genocide », BBC News (14 août 2004), [en ligne] http://news.bbc.co.uk/2/hi/africa/3565938.stm, page consultée le 10 décembre 2012.

39. Nadja Vuckovic, loc. cit., p. 775.

40. Ingolf Diener, op. cit., p. 121.

41. David Olusoga, op. cit.

42. Ibid.

43. Ingolf Diener, op. cit., p. 119.

44. Ibid.

45. Ibid., p. 124. 
individus issus de ces deux peuples sont par ailleurs dorénavant contrôlés par un système de passeports et de permis de travail ${ }^{46}$. Ce système sera perpétué après 1915 à travers la conquête de l'ancienne colonie par les Sud-Africains ${ }^{47}$.

Toutefois, même sans que survienne le génocide, les sociétés herero et nama traditionnelles auraient fini par disparaître, condamnées par l'avancée inexorable des politiques de Leutwein déjà en voie de dominer la région en $1904^{48}$. Là où le génocide opère réellement un bouleversement, c'est au niveau démographique : les bantouphones cessent d'être le groupe ethnolinguistique majoritaire de la région, titre qui revient d'office aux Ovambo. Ce groupe détient d'ailleurs actuellement la prédominance numérique en Namibie. Les descendants des Herero et Nama vivent donc aujourd'hui en Namibie des situations de marginalisation et de précarité économique directement liées aux suites du génocide ${ }^{49}$. C'est aussi à cette époque que se sont cristallisées les profondes disparités économiques entre les populations d'origine africaine et d'origine européenne en Namibie, disparités qui sont encore d'actualité50.

\section{MÉmoIRES DU GÉNOCIDE}

Ce n'est qu'au début des années 2000, alors qu'approche le centenaire de la guerre herero-allemande, que les descendants des Herero et des Nama commencent à faire valoir la mémoire du " génocide oublié ». Ils sont alors les seuls à entretenir le souvenir de cette tragédie. En Allemagne, la période coloniale est largement reléguée aux oubliettes mémorielles, alors qu'en Namibie, c'est l'indépendance, obtenue en 1990 par la SWAPO (South West Africa Political Organisation) de Sam Nujoma - président jusqu'en 2005 - qui tient lieu de récit fondateur. Penchons-nous donc maintenant sur ces deux constructions mémorielles.

L'AMNÉSIE ALLEMANDE

«Le sombre chapitre d'une lointaine histoire commune ${ }^{51} »$ : c'est en ces termes vaguement euphémiques que Roman Herzog, président fédéral allemand, caractérise les événements du début du siècle dans l'Afrique allemande du Sud-Ouest lors de son passage dans la capi-

46. Ibid.

47. Ibid., p. 155.

48. Ibid., p. 123.

49. Bernard Lugan, op. cit., p. 244.

50. Ingolf Diener, op. cit., p. 124.

51. Johannes Wendt, loc. cit., p. 64. 
tale namibienne Windhoek en $1998^{52}$. Sombre, ce chapitre semble en même temps lointain pour les Allemands.

De tels propos découlent directement de la mémoire collective entretenue par les Allemands vis-à-vis de la période coloniale de leur histoire nationale. En effet, comme l'exprime Johannes Wendt, «les crimes coloniaux allemands par lequel s'est ouvert, surtout en Chine, en Afrique du Sud-ouest et de l'Est, le siècle du génocide, sont autant de pages blanches ${ }^{53}$. " Pourquoi une telle amnésie? Tout d'abord en raison du discours historique officiel, qui minimise grandement l'implication allemande dans le phénomène colonial. L'empire colonial allemand a effectivement connu une vie relativement courte : ses timides débuts, qui suivent l'unification du pays, se font en $1871^{54}$. Cette entrée dans le grand jeu expansionniste peut paraître assez tardive, surtout lorsqu'on compare l'empire colonial allemand à celui d'une puissance comme la France, qui débute son âge impérialiste dès le XVle siècle. De plus, son démantèlement en 1919 - scellé par le traité de Versailles - termine assez rapidement cette expérience coloniale; la future Namibie elle-même est conquise par les troupes sud-africaines dès 191555. Ce demi-siècle de colonialisme est ainsi balayé du revers de la main dans le discours officiel comme ne constituant pas une « vraie » période coloniale en raison de sa brièveté : la mémoire collective allemande se considère par conséquent comme étant exempte de la «tache » du colonialisme, surtout lorsqu'elle se place dans une perspective comparatiste avec la France ou la Grande-Bretagne ${ }^{56}$.

Il ne s'agit cependant pas uniquement d'une entreprise de minimisation : une certaine désinformation est également identifiable dans l'historiographie. En effet, les quelques ouvrages publiés au sujet du colonialisme allemand avant les années 1980 s'articulent principalement autour du vieux mythe de l'Occidental civilisateur ${ }^{57}$, allant jusqu'à dépeindre les méthodes coloniales allemandes comme étant plus respectueuses que celle des autres métropoles. Essentiellement, en Allemagne, on semble considérer le colonialisme comme l'affaire des autres : bien que les documents officiels traitant de ce phénomène discutent bel et bien des atrocités commises en Afrique par les pouvoirs coloniaux, les exemples donnés sont limités aux victimes d'autres puissances.

\section{Ibid.}

53. Ibid., p. 57.

54. Ingolf Diener, op. cit., p. 70.

55. Ibid., p. 147.

56. Johannes Wendt, loc. cit., p. 60.

57. Jurgen Zimmerer, « Colonial Genocide : The Herero and Nama War (1904-08) in German South West African and its Significance ", dans Dan Stone, dir., The historiography of genocide, New York, Palgrave Macmillan, 2008, p. 335. 
Ainsi, les brochures éducatives mises gratuitement à la disposition des enseignants par le Bureau fédéral d'éducation politique illustrent les crimes coloniaux par des photographies montrant des victimes mutilées sous le joug du Congo Belge en raison d'une production insuffisante de caoutchouc ${ }^{58}$. Cet effacement de la période coloniale allemande dans le discours mémoriel, perçue comme trop courte pour avoir été le théâtre d'horreurs de ce calibre, remonte au contexte de l'après-guerre, alors que l'Allemagne entre dans une période de prospérité doublée de l'anxiété générale caractérisant le début de la Guerre froide ${ }^{59}$.

Une autre cause de cette amnésie réside dans la possibilité d'établir des parallèles entre le génocide herero et l'Holocauste ${ }^{60}$. Cette épineuse question étant suffisamment complexe pour constituer l'objet d'une étude entière, nous n'examinerons que l'effet que cette ressemblance a pu avoir sur la mémoire collective allemande. À bien des égards, l'Holocauste est aujourd'hui un élément central des mémoires collectives en Occident. Sa reconnaissance est même devenue un prérequis pour toute nation souhaitant intégrer l'Union européenne : c'est le fameux « critère mémoriel de Copenhague ${ }^{61}$ », qui tend à hiérarchiser les mémoires des crimes de masse en positionnant l'Holocauste au sommet de l'échelle de l'horreur. Pourtant, malgré la prévalence généralisée de la Shoah en tant que lieu de mémoire commun de la Seconde Guerre mondiale, la question de ses causes profondes s'éclipse, comme l'avait pressenti Hannah Arendt, face à la fascination morbide suscitée par les méthodes employées dans l'extermination des juifs.

Ainsi, dans les mémoires collectives européennes, le génocide juif tient de «l'accident » historique : c'est un dérapage, une erreur de la nature, un évènement qui n'aurait jamais dû survenir ${ }^{62}$ et qui est principalement attribuable à la folie d'un homme, Adolf Hitler, et à ses «fantasmes de guerre raciale ${ }^{63} »$. Cette mémoire de la Shoah a l'avantage d'être plus facile à assumer pour la collectivité qui doit en supporter la responsabilité historique : elle entraîne réparations et reconnaissance, certes, mais ne suscite aucune remise en question du caractère national allemand. Comme le formule expertement Jürgen Zimmerer, «si Hitler n'était qu'un déraillement survenu sur un chemin autrement positif, alors ces 12 «sombres années» peuvent facilement être exorcisées de l'histoire nationale allemande ${ }^{64}$. »

58. Johannes Wendt, loc. cit., p. 61.

59. Ibid., p. 62.

60. Reinhart Kössler, «From Genocide to Holocaust? Structural Parralels and Discursive Continuities », Africa Spectrum, vol. 40, n² (2005), p. 315.

61. Emmanuel Droit, loc. cit., p. 105.

62. Jürgen Zimmerer, loc. cit., p. 324.

63. Ibid., p. 334. Traduction libre.

64. Ibid. 
Insérons maintenant le génocide herero et nama dans ce portrait : l'image obtenue est discordante. Si l'Holocauste n'est rien d'autre qu'un accroc dans la toile historique de l'Europe, comment expliquer les parallélismes troublants entre ces deux génocides ${ }^{65}$ ? C'est en grande partie pour éviter de devoir répondre à cette question lourde d'implications que le génocide herero a connu une telle occultation en Allemagne. En effet, si la mémoire du génocide herero rencontrait un écho au sein du public, on assisterait assurément à une réévaluation de la responsabilité historique germanique dans l'Holocauste, dont les stigmates mémoriels sont déjà lourds à porter. Toutefois, il serait faux d'affirmer que l'Allemagne n'a d'aucune façon commencé l'anamnèse de sa période coloniale, bien que la discussion critique sur ce sujet ait été pour le moment circonscrite aux milieux intellectuels ${ }^{66}$ : depuis le centenaire du génocide herero, des auteurs allemands comme Reinhart Kössler, Jürgen Zimmerer ou Henning Melber ont produit de nombreuses réflexions explorant l'histoire coloniale allemande ${ }^{67}$.

L'idée de la présence d'une continuité entre génocides herero et juif a été particulièrement visitée par ces auteurs, bien que d'autres, comme Robert Gerwarth et Stephan Malinowski, contestent cette idée comme étant de nature trop conjecturale ${ }^{68}$. On assiste également à une révision de l'idée du Sonderweg, le « chemin particulier » du racisme allemand qui aurait amené la transgression du tabou culturel de l'extermination ${ }^{69}$, ainsi qu'à un exercice de réécriture de l'histoire de la Shoah dans une perspective englobant l'expérience coloniale allemande ${ }^{70}$. La mémoire collective européenne, en revanche, n'est que marginalement touchée par ces efforts. Dans les strates populaires, on trouve peu de traces du génocide herero ailleurs que sur les sites Internet des organismes dédiés à la réparation des crimes coloniaux, comme Colonialism Reparation ${ }^{71}$, organisme humanitaire d'origine italienne, ou Peace Pledge Union ${ }^{72}$, regroupement pacifiste britannique.

En somme, si la mémoire allemande du génocide herero est caractérisée par l'oubli, c'est tout d'abord en raison d'un important discours de minimisation de la période coloniale allemande. II s'agit également d'un débat soigneusement évité en raison de son lien avec l'Holocauste: ouvrir le dossier du génocide herero revient également à rouvrir celui des origines profondes

\section{Ibid.}

66. Johannes Wendt, loc. cit., p. 70.

67. Ibid., p. 70.

68. Robert Gerwarth et Stephan Malinowski, loc. cit., p. 143.

69. Ibid., p. 151.

70. Ibid.

71. Colonialism Reparation, « Namibie-Allemagne » (2012), [en ligne]www.colonialismreparation.org, page consultée le 20 décembre 2012.

72. Peace Pledge Union, « Namibia 1904 », Talking about genocide, [en ligne]www.ppu.org.uk, page consultée le 20 décembre 2012. 
du génocide juif. Dans les deux cas, c'est le refus de la part des Allemands de réinscrire - à la lumière de ces deux tragédies - la responsabilité de leur pays dans une perspective historique de longue durée qui explique cette lacune mémorielle.

\section{Un hÉRItAGe UNILATÉral : Herero et Namibie}

Dans la section précédente, nous avons abordé la mémoire allemande comme un ensemble relativement homogène. Toutefois, il est impossible de traiter de la Namibie de la même façon : si la mémoire du génocide y a été conservée par les descendants du génocide, en revanche, la société namibienne dans son ensemble est loin d'avoir adhéré à ce récit. Malgré ce qu'on pourrait croire, le génocide herero et nama a en effet connu une occultation poussée en Namibie : même les plus anciens manuels d'histoire utilisés au pays ne mentionnent ni l'ordre d'extermination de Von Trotha, ni les camps de concentration, ni les morts survenues dans le désert de l'Omaheke ${ }^{73}$.

L'Afrique allemande du Sud-Ouest est au contraire dépeinte comme un paradis colonial ensoleillé et harmonieux, élevée aux nues tel un âge d'or perdu ${ }^{74}$. II s'agit d'une représentation en partie façonnée à l'intention des touristes sud-africains, que les plages sablonneuses de Namibie attirent en grand nombre chaque année ${ }^{75}$. À Swakopmund, phare touristique et ancien emplacement d'un des plus grands camps de concentration herero, tout est d'ailleurs à saveur allemande : la cuisine, les noms des commerces, et même les souvenirs, dont l'es-

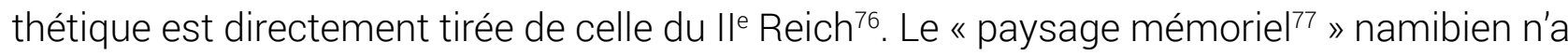
également retenu que très peu de traces physiques du génocide : l'emplacement précis du camp de Swakopmund a par exemple été perdu, si bien que l'endroit où les descendants des victimes déposent des fleurs en mémoire de leurs aïeux n'est qu'une approximation ${ }^{78}$.

On peut tout d'abord attribuer cette occultation au système de domination lié à l'apartheid et imposé par la conquête sud-africaine ${ }^{79}$; mais il faut, en outre, la replacer également dans une optique de concurrence mémorielle au sein de la Namibie. En effet, un évènement particulier de l'histoire coloniale supplante tous les autres dans la mémoire collective namibienne:

73. David Olusoga, op. cit.

74. Ibid.

75. Ibid.

76. Ibid.

77. Reinhart Kössler, "Facing a Fragmented Past : Memory, Culture and Politics in Namibia », Journal of Southern African Studies, vol. 33, n 2 (juin 2007), p. 367.

78. David Olusoga, op. cit.

79. Ingolf Diener, op. cit., p. 165. 
il s'agit de la libération du pays en $1990^{80}$. La raison de la prééminence mémorielle de cet évènement s'explique d'une façon évidente, puisque le parti qui se veut l'artisan de cette libération, la SWAPO (South-West African People's Organisation), est encore au pouvoir aujourd'hui. Qui plus est, l'homme à la tête du mouvement de libération, Sam Nujoma, a dirigé le pays dès son accession à l'indépendance jusqu'en 2005.

La mémoire du colonialisme mise en avant est donc bien entendu celle de cette victoire qui rehausse l'image du parti au pouvoir : c'est une mémoire triomphaliste, centrée autour du personnage du fier soldat de la libération ${ }^{81}$. Cette mémoire trouve d'ailleurs son incarnation dans le monument Heroes' Acre, inauguré à Windhoek en 2002. On peut y admirer la statue de bronze d'un soldat accompagnée d'un obélisque et d'une frise ${ }^{82}$. Le monument, réalisé par une firme nord-coréenne, se veut également le symbole du projet politique de la SWAPO : une nation unie, militaire, socialiste ${ }^{83}$. Dans ce contexte, on peut comprendre pourquoi la mémoire du génocide herero, qui est caractérisée par une certaine exclusivité ethnique et par un caractère victimaire, n'a pas été adoptée d'emblée par le gouvernement namibien. En effet, mettre l'emphase sur les souffrances d'un groupe particulier tend à favoriser les dissensions, ce qui va directement à l'encontre d'un tel projet; de plus, une mémoire liée au statut de victime entrerait en contradiction avec l'image du Namibien vainqueur et triomphant mise en avant par la SWAPO.

Jusqu'au début des années 2000, la mémoire du génocide subsiste donc surtout dans la tradition herero : il s'agit même du vecteur central autour duquel s'articule l'entièreté de leur identité ${ }^{4}$. De nombreux descendants des survivants des camps de concentration sont en effet encore vivants aujourd'hui; certains possèdent même encore les plaques de métal numérotées que leurs parents étaient forcés de porter ${ }^{85}$. Toutefois, une fois entamé le troisième millénaire, des changements s'effectuent alors que le centenaire du génocide approche : on voit réapparaître sur la place publique la question du génocide lors d'évènements comme le Jour des Herero, fête annuelle célébrant l'enterrement de Samuel Maharero ${ }^{86}$. Ainsi, en 2002, le controversé chef herero Kuaima Riruako déclare durant une allocution effectuée à cette

80. Reinhart Kössler, loc. cit., juin 2007, p. 369.

81. Ibid.

82. Ibid.

83. Ibid., p. 370.

84. Ibid., p. 378.

85. Ibid.

86. Henning Melber, « How to Come to Terms with the Past : Re-visiting the German Colonial Genocide in Namibia », Africa Spectrum, vol. 40, n 1 (2005), p. 141. 
occasion que les revendications territoriales effectuées en lien avec le passé colonial ne concernent que les Herero ${ }^{87}$.

Cette déclaration souligne bien le conflit de mémoires qui s'esquisse en Namibie depuis le début de l'extériorisation de la mémoire du génocide en dehors du cadre restreint de la communauté herero. Tout d'abord, il importe de mentionner la réaction des membres de la communauté germanophone namibienne : ces commémorations enflamment chez eux un débat sur le génocide, largement nié. Ceux-ci interprètent notamment la décision de Von Trotha de pousser les Herero à fuir dans le désert de l'Omaheke comme un geste de pitié et non d'extermination; ce type de discours reste toutefois presque exclusif à ce groupe d'origine allemande ${ }^{88}$. Les réactions au discours de Riruako sont également virulentes chez les groupes d'origine africaine : les descendants des Nama, des Damara et des San, dont les ancêtres ont également été touchés par le colonialisme, s'insurgent contre ce qu'ils considèrent être la monopolisation du statut de victime par les Herero ${ }^{89}$.

Ces protestations se poursuivent jusqu'en 2004, tandis que des groupes herero mettent en scène de nombreux évènements commémoratifs entourant l'année centenaire du génocide. Ceux-ci débutent avec la célébration du commencement de la guerre contre les Allemands le 10 janvier, atteignent leur apogée avec l'évènement Ohakamari de Waterberg lors de l'anniversaire de la bataille du même nom, et se terminent avec la commémoration de l'ordre d'extermination le 2 décembre ${ }^{90}$. Durant ces évènements, qui consacrent l'ouverture de la mémoire collective namibienne au récit du génocide herero, les représentants de ce groupe sont accusés entre autres choses d'abuser de leur « authenticité biologique ${ }^{91}$ » pour s'arroger le statut de victime. Cette séparation identitaire qui s'effectue entre les Herero, victimes d'un génocide éponyme, et le reste de la population, qui ne peut prétendre à ce titre, tend ainsi à créer une division et à favoriser la concurrence des identités victimaires ${ }^{92}$. Les questions mémorielles liées au génocide Herero en Namibie sont donc loin de faire l'unanimité, et brossent le portrait d'une nation aux identités multiples et conflictuelles.

Bref, pour les Namibiens, la mémoire du génocide Herero s'articule également autour d'un certain oubli, effectué dans un premier temps en raison de la poursuite du système d'oppression coloniale. Dans un deuxième temps, le génocide doit son occultation à la présence dans les mémoires d'un autre évènement lié au colonialisme et politiquement plus favorable

\section{Ibid.}

88. Reinhardt Kössler, loc. cit., juin 2007, p. 377.

89. Henning Melber, loc. cit., p. 141.

90. Reinhardt Kössler, loc. cit., juin 2007, p. 378.

91. Henning Melber, loc. cit., p. 141.

92. Ibid. 
au parti au pouvoir, celui de la libération de la nation en 1990. De plus, bien que la mémoire du génocide cesse, en 2004, d'être l'apanage de la seule communauté herero, l'acceptation du récit est loin d'être unanime. La communauté germanophone, attaquée dans sa mémoire nostalgique du colonialisme, préfère nier le caractère génocidaire des évènements de 19041908. D'autres groupes revendiquant également le statut de victime s'offusquent de sa monopolisation par les Herero, complétant le tableau d'une situation complexe placée sous le signe d'une concurrence multiple des mémoires.

\section{RENCONTRE ET REVENDICATIONS : DÉMARCHES HERERO, INCONFORT ALLEMAND}

L'extériorisation de la mémoire du génocide telle qu'entretenue par les Herero ne s'arrête pas à la seule Namibie, mais se poursuit à travers des demandes de réparation adressées aux Allemands. Ces démarches, loin d'être accueillies favorablement par la nation allemande, provoquent la rencontre gênante de deux mémoires diamétralement opposées.

C'est dans les années suivant l'indépendance de la Namibie que le Conseil pour les Dédommagements au Peuple Herero est créé par Kuaima Riruako et par le professeur Mburuma Kerina, tous deux descendants directs de victimes du génocide ${ }^{93}$.

L'organisme, à l'origine de la majorité des revendications des Herero, débute ses réclamations en 2001, entamant des démarches auprès de l'Allemagne pour obtenir une juste réparation pour les infractions commises envers les droits de l'homme durant la période coloniale. En revanche, c'est une Allemagne relativement peu encline à négocier que rencontrent les membres du Conseil : comme il a été expliqué plus haut, la mémoire du colonialisme entretenue par la nation allemande est basée sur une désaffectation vis-à-vis de celle-cip4.

En 2001, Mburuma Kerina décide toutefois de mettre un peu de pression sur l'ancienne puissance coloniale pour que celle-ci cesse de minimiser son rôle et reconnaisse autre chose qu'une « responsabilité particulière ${ }^{95}$ » dans les crimes coloniaux commis en Namibie. II dépose ainsi, en juin 2001, une plainte officielle au nom du Conseil auprès de la Haute Cour du District de Columbia, tribunal habilité par le droit américain et international à imposer des sanctions pour les crimes historiques ${ }^{96}$. Son but dans cette entreprise est double, d'après Nadja Vuckovic : non seulement le Conseil souhaite obtenir une reconnaissance internationale des crimes commis à l'endroit des Herero dans une perspective mémorielle, mais il cherche également une réelle compensation pour les bénéfices économiques que l'Alle-

93. Nadja Vuckovic, loc. cit., p. 776.

94. Johannes Wendt, loc. cit., p. 60.

95. Ibid.

96. Nadja Vuckovic, loc. cit., p. 776. 
magne a retirés du génocide à travers l'expropriation, la confiscation des biens et l'usage de la main-d'œuvre détenue dans les camps de concentration.

Le Conseil milite donc en faveur d'une réparation monétaire qui permettrait aux Hereros de racheter leurs terres, ce qui contribuerait à remédier à leur situation de pauvreté et de marginalisation issue des suites du génocide ${ }^{97}$. II s'agit en effet de la raison d'être des réparations mémorielles : comme l'écrit Bogumil Jewsiewicki, « sans prétendre restaurer à l'identique le passé, les réparations visent à replacer la victime ou ses descendants - personnes ou communautés - là où elles auraient dû être si elles avaient conservé le contrôle de leurs biens et de leur cadre de vie matérie|98.

En 2004, une première réponse de l'Allemagne est effectuée à l'occasion des célébrations du centenaire, lorsque Wolfgang Massing, ambassadeur allemand en Namibie, prend la parole lors de la commémoration de l'attaque d'Okahandja. Excluant toute réparation monétaire malgré la plainte, il exprime toutefois un profond regret de la part des Allemands à l'égard du passé $^{99}$. L'intervention la plus remarquée est toutefois celle de la ministre au Développement et à la Coopération économique de l'Allemagne, Heidemarie Wieczorek-Zeul, qui accepte l'invitation des Herero de représenter son pays lors de la cérémonie commémorant la bataille de Waterberg. À cette occasion, Wieczorek-Zeul prend la parole devant 4000 spectateurs, qualifiant les actes perpétrés par le général von Trotha de génocide ${ }^{100}$, particulièrement le fameux ordre d'extermination; en larmes devant un portrait de Samuel Maharero, elle invite ensuite les Herero à porter le deuil et à pardonner aux Allemands « dans l'esprit d'un Notre Père collectif ${ }^{101} »$ et dans le but qu'un jour leurs deux nations puissent espérer se réconcilier ${ }^{102}$.

L'impact de ces déclarations est considérable. Au départ, celles-ci sont accueillies très favorablement, mais, pour le Conseil de Dédommagement, elles sont de loin insuffisantes. En effet, elles ne font allusion qu'aux actes de Von Trotha, sans aucune mention des camps de concentration, de l'exploitation et de la déshumanisation qui ont suivi la guerre proprement dite $^{103}$. Également, l'analyse de la teneur de ces propos révèle un message beaucoup moins apologétique qu'il n'y paraît : l'esprit d'un « Notre Père collectif » n'implique-t-il pas une double responsabilité? Comme le souligne Johannes Wendt, «si l'on s'en tient au texte biblique -

\section{Ibid.}

98. Bogumil Jewsiewicki-Koss, " Héritages et réparations en quête d'une justice pour le passé et le présent », Cahiers d'études africaines, n 173-174 (2004), p. 8.

99. [s. a.], " Germany regrets Namibia's 'genocide' », BBC News, (12 janvier 2004), [en ligne] http://news.bbc.co.uk/2/hi/africa/3388901.stm, page consultée 20 décembre 2012.

100. [s. a.], loc. cit., 14 août $2004 .$.

101. Johannes Wendt, loc. cit., p. 65.

102. David Olusoga, op. cit.

103. Ibid. 
«Pardonne-nous nos offenses comme nous pardonnons aussi à ceux qui nous ont offensés» - la phrase implique une macabre excuse ${ }^{104}$. » De plus, la réconciliation offerte par WieczorekZeul prend vite l'allure, au sein des communautés herero, de l'achat de leur silence au sujet du génocide : elle propose en effet au nom de l'Allemagne un versement de 20 millions, versé prioritairement aux descendants des victimes en échange de leur pardon ${ }^{105}$. II s'agit d'une somme substantielle, mais ce n'est que le centième du montant revendiqué par le Conseil, qui a été calculé en fonction des gains faits par les Allemands grâce à la main d'œuvre carcérale herero. De plus, cette offre a été interprétée comme insultante et immorale, les descendants des victimes considérant qu'il leur revient de donner leur pardon lorsque les plaies se seront refermées, et qu'il ne convient pas à l'Allemagne de tenter de l'acheter ${ }^{106}$.

Cette offre de réparation, si on l'interprète à l'aune de la construction mémorielle allemande, prend par ailleurs l'allure étrange d'une maladroite tentative d'achat d'une prolongation de l'amnésie allemande au sujet de sa période coloniale : l'intervention de Wieczorek-Zeul, fort médiatisée en Afrique, ne connaît pas le même retentissement en Europe. Le statu quo y est par conséquent relativement intact, et, en cas d'une réponse positive des Herero face à cette offre, tout conflit mémoriel susceptible d'engendrer un débat public aurait été étouffé.

L'année suivante, lors de sa visite en Allemagne, Hifikepunye Lucas Pohamba, nouveau président namibien, décline une offre de réparations semblable préparée à son attention ${ }^{107}$. Les relations entre les autorités de Windhoek et de Berlin, villes par ailleurs jointes par un jumelage depuis 2000, semblent avoir atteint un point mort ${ }^{108}$. Durant les deux années suivant le centenaire du génocide et le rejet de l'offre de Wieczorek-Zeul, l'Allemagne semble durcir ses positions par rapport au génocide, étant toujours peu encline à étendre l'appellation de «génocide» à l'ensemble des mesures prises à l'égard des Herero et des Nama en Afrique allemande du Sud-Ouest ${ }^{109}$. D'après Esther Utijua, membre du Conseil de Dédommagement pour le peuple Herero, I'Allemagne adopte une attitude vague, presque hostile, et refuse un dialogue approfondi1 ${ }^{10}$. En 2009, le dossier des réparations demandées par les Herero est d'ailleurs toujours gelép ${ }^{11}$.

104. Johannes Wendt, loc. cit., p. 65.

105. Ibid., p. 73.

106. Ibid., p. 74.

107. Ibid., p. 65.

108. Ibid.

109. Ibid., p. 76.

110. Peter Biles, loc. cit.

111. Nicolas Champeaux, "Le peuple herero demande réparation », RFI, (26 novembre 2009), [en ligne] http://www.rfi.fr/contenu/20091126-le-peuple-herero-demande-reparation, page consultée le 20 décembre 2012. 
Cependant, en 2011, l'Allemagne pose de nouveau un geste à l'égard des Herero : le 30 septembre, I'hôpital de la Charité à Berlin accueille une délégation namibienne et lui restitue 20 crânes de victimes du génocide ${ }^{112}$. Ces crânes, prélevés dans les camps de concentration d'Afrique allemande du Sud-ouest, avaient été acheminés vers l'Allemagne pour y être étudiés. Au début du siècle dernier, Berlin est en effet un centre de recherches anthropologiques important ainsi qu'une des figures de proue de l'élaboration du racisme scientifique ${ }^{113}$. Pour Johanna Ka-A-Jipara, un des membres de la délégation interviewé pour l'occasion, cet évènement est bouleversant de par sa charge symbolique collective et personnelle : sa grandmère fut une survivante des camps de concentration, et le récit qu'elle lui a légué constitue une part importante de son identité 114 .

Des nuages viennent cependant obscurcir ce qui semble être le premier geste de bonne volonté de la part des officiels allemands depuis huit ans : la nouvelle ministre allemande déléguée aux Affaires Étrangères, Cornelia Pieper, est huée par des manifestants lors de son discours dans lequel elle réclame à son gouvernement « des réparations et une reconnaissance plus large des crimes de l'empire colonial allemand ${ }^{115}$. » En dépit des manifestations hostiles qu'elle a rencontrées - et qui témoignent encore et toujours de l'inconfort allemand face à la mémoire du génocide herero, nous pouvons malgré tout voir en cette intervention un signe que la mémoire collective allemande s'engage, petit à petit, sur la voie d'une véritable anamnèse de son colonialisme.

Le résultat de cette anamnèse sera-t-il positif, ou sera-t-il marqué par un rejet de l'Autre? Là réside le nœud de l'affaire. En somme, à l'heure actuelle, l'inconfort caractérisant la rencontre entre les Hereros et l'Allemagne, qui reste le principal partenaire économique de la Namibie $^{116}$, reste vif. La plainte déposée par Kerina reste à ce jour sans suite. Le gouvernement namibien, qui bénéficie par ailleurs d'une aide au développement de près de 14 milliards d'euros par année de la part de l'Allemagne ${ }^{117}$, a longtemps refusé d'appuyer les démarches revendicatrices des Herero, préférant prioriser l'unité nationale118. Toutefois, depuis 2007, l'Assemblée nationale de Windhoek approuve ces demandes de dédommagements à l'una-

112. [s. a.], " Un siècle après leur extermination, l'Allemagne restitue des crânes aux Hereros et Namas », RFI, (1er octobre 2011), [en ligne] http://www.rfi.fr/afrique/5min/20111001-siecle-apres-leur-extermination-allemagne-restitue-cranes-peuples-herero-nama, page consultée le 20 décembre 2012.

113. Ibid.

114. Ibid.

115. Ibid.

116. Peter Biles, loc. cit.

117. Nadja Vuckovic, loc. cit., p. 776.

118. Henning Melber, loc. cit., p. 142. 
nimité, montrant une évolution de la conscience collective vers une acceptation élargie de la mémoire du génocide.

\section{ConClusion}

Que doit-on retenir de ce large portrait de la situation du conflit mémoriel où Herero et Allemands peinent à établir le dialogue? Premièrement, il est manifeste que le génocide de 1904-1908 est l'élément de base de l'identité Herero, le cœur d'où émanent leur mémoire et leur expérience du monde contemporain. Deuxièmement, en parallèle de l'hypermnésie vécue par les Herero, nous pouvons constater son absence mémorielle chez les Allemands, absence liée à un phénomène de minimisation de l'impact du colonialisme de cette nation en Afrique. Troisièmement, il paraît évident que la Namibie est moins unie qu'elle ne le paraît face à son "passé fragmenté119 ». Finalement, nous avons pu déterminer que le moment clé qui souligne le commencement du dialogue mémoriel entre l'Allemagne et la Namibie est l'allocution fortement médiatisée de la ministre allemande Heidemarie Wieczorek-Zeul à l'occasion de la commémoration du centenaire de la bataille de Waterberg en 2004. Bien que le dialogue reste intermittent et tendu, la restitution en 2011 de 20 crânes de victimes du génocide témoigne d'un début d'ouverture chez les Allemands. Le portrait mémoriel ainsi formé répond à la question que nous nous étions posée au début de ce texte : comment se construisent les modalités de l'oubli et de la mémoire chez les Namibiens et les Allemands, et comment s'articule leur rencontre? Nous pouvons affirmer avoir vérifié l'ensemble de nos hypothèses.

Cependant, un des aspects soulevés au cours de notre recherche ne reste pas totalement exploré : il s'agit de l'analyse du génocide Herero et du génocide juif dans une perspective de continuité, et de l'impact que la création d'une telle mémoire en Europe pourrait avoir sur les constructions mémorielles existantes. Pensons notamment à l'Europe de l'Est, où la mémoire de la Shoah est relativement mal vécue en raison de sa concurrence avec la mémoire des crimes de Staline ${ }^{120}$. Quels changements seraient amenés par l'introduction d'un élément africain dans le continuum mémoriel? II est possible qu'une telle globalisation de la mémoire amène également de nouvelles revendications comme celles des Herero, qui ne sont ni les premiers, ni les derniers à chercher réparations pour les crimes du passé. Car si le $X X^{e}$ siècle a été celui de la violence, le XXle est celui des réparations : sans être faciles à gérer, elles sont assurément nécessaires à la mise en place harmonieuse de la nouvelle communauté internationale.

119. Reinhardt Kössler, loc. cit., juin 2007, p. 361.

120. Emmanuel Droit, loc. cit., p. 101. 


\section{SOURCES ET BIBLIOGRAPHIE}

[s. a.]. «A bloody history: Namibia's colonisation ». BBC News (29 août 2001), [en ligne]http://news. bbc.co.uk/2/hi/africa/1514856.stm (page consultée le 10 décembre 2012).

[s. a.]. « Germany regrets Namibia's 'genocide' ». BBC News, (12 janvier 2004), [en ligne] http://news. bbc.co.uk/2/hi/africa/3388901.stm, page consultée 20 décembre 2012.

[s. a.]. « Germany admits Namibia's genocide ». BBC News (14 août 2004), [en ligne] http://news.bbc. co.uk/2/hi/africa/3565938.stm, page consultée le 10 décembre 2012.

[s. a.]. « Un siècle après leur extermination, l'Allemagne restitue des crânes aux Hereros et Namas ». RFI, (1 ${ }^{\text {er }}$ octobre 2011), [en ligne] http://www.rfi.fr/afrique/5min/20111001-siecle-apres-leur-extermination-allemagne-restitue-cranes-peuples-herero-nama, page consultée le 20 décembre 2012.

AKBAR, Noor. «How should we define genocide? ». Human Dignity and Humiliation Studies, [en ligne] www.humiliationstudies.org, page consultée le 15 décembre 2012.

BILES, Peter. « Unfinished Business for Namibia's Herero ». BBC News (19 janvier 2006), [en ligne] http://news.bbc.co.uk/2/hi/africa/4623516.stm (page consultée le 19 novembre 2012).

CHAMPEAUX, Nicolas. « Le peuple herero demande réparation ». RFI, (26 novembre 2009), [en ligne] http://www.rffi.fr/contenu/20091126-le-peuple-herero-demande-reparation, page consultée le 20 décembre 2012.

Colonialism Reparation. « Namibie-Allemagne ». (2012), [en ligne] www.colonialismreparation.org, page consultée le 20 décembre 2012.

COQ, Christian, dir. Travail de mémoire 1914-1998. Une nécessité dans un siècle de violence. Paris, Autrement, 1999, $270 \mathrm{p}$.

DIENER, Ingolf. Namibie, une histoire, un devenir. Paris, Karthala, 2000, 382 p.

DROIT, Emmanuel. « Le Goulag contre la Shoah : Mémoires officielles et cultures mémorielles dans l'Europe élargie ». Vingtième Siècle. Revue d'histoire, n 94 avril-juin 2007, p. 101-120.

FERRO, Marc, dir. Le livre noir du colonialisme. XVIème-XXlème siècle : de l'extermination à la repentance. Paris, Robert Laffont, 2003, 843 p.

GERWARTH, Robert et Stephan MALINOWSKI. "L'antichambre de l'holocauste? À propos du débat sur les violences coloniales et l'extermination nazie ». Vingtième siècle. Revue d'histoire, vol 3, n’ 99 (2008), p. 143-159.

GEWALD, Jan-Bart. «The Road of the Man Called Love and the Sack of Sero: The Herero-German War and the Export of Herero Labour to the South African Rand ». The Journal of African History, vol 40, nº 1 (1999), p. 21-40. 
ILIFFE, John. Africans : the history of a continent. Cambridge, Cambridge University Press, 2007 (1995), $388 \mathrm{p}$.

JEWSIEWICKI-KOSS, Bogumil. « Héritages et réparations en quête d'une justice pour le passé et le présent ». Cahiers d'études africaines, nº 173-174, 2004, p. 7-24.

KÖSSLER, Reinhart. « From Genocide to Holocaust? Structural parallels and discursive continuities ». Africa Spectrum, vol 40, nº 2 (2005), p. 309-317.

KÖSSLER, Reinhart. «Facing a Fragmented Past: Memory, Culture and Politics in Namibia ». Journal of Southern African Studies, vol 33, nº 2 (juin 2007), p. 361-382.

LUGAN, Bernard. Histoire de l'Afrique du Sud de l'Antiquité à nos jours. Paris, Perrin, 1986, p. 272.

OLUSOGA, David. Namibia: Genocide and the Second Reich. Enregistrement vidéo, BBC Bristol, Bristol, 2005. 1 vidéodisque : 58 minutes, son, coul. DVD.

MELBER, Henning. " How to come to terms with the past : Re-visiting the German colonial genocide in Namibia ». Africa Spectrum, vol. 40, nº 1 (2005), p. 139-148.

Peace Pledge Union. «Namibia 1904 ». Talking about genocide, [en ligne] www.ppu.org.uk, page consultée le 20 décembre 2012.

STONE, Dan, dir. The historiography of genocide. New York, Palgrave Macmillan, 2008, 400 p.

WENDT, Johannes. «L'anamnèse du colonialisme allemand ». L'homme et la société, nº 175 (2001), p. 57-80. 